\title{
Dental approach to erosive tooth wear in gastroesophageal reflux disease
}

\author{
Ayse Dundar ${ }^{1}$, Abdulkadir Sengun ${ }^{2}$
}

1. Department of Restorative Dentistry, School of Dentistry, University of Abant Izzet Baysal, Bolu, Turkey

2. Department of Restorative Dentistry, School of Dentistry, University of Kirikkale, Kirikkale, Turkey

\begin{abstract}
Background: The duration of gastro-esophageal reflux disease (GERD), the frequency of reflux, the $\mathrm{pH}$ and type of acid, and the quality and quantity of saliva affect the severity of dental erosion due to GERD.

Objective: To summarize the diagnostic protocol and treatment of dental erosion due to GERD.

Methods: A Medline literature search was performed to identify articles associated with a dental approach to GERD.

Results: The dental professional must carry out a diagnostic protocol, which includes collecting data on the patient's medical and dietary history, occupational/recreational history, dental history, and oral hygiene methods. Intraoral, head and neck, and salivary function examinations should be performed to expose the dental implications of GERD symptoms.

Conclusion: Diagnosing the cause of erosive tooth wear can help prevent further damage. Patients must be informed about how to prevent GERD.
\end{abstract}

Keywords: Dental erosion, gastro-esophageal reflux disease

African Health Sciences 2014; 14(2):481-486

DOI: http://dx.doi.org/10.4314/ahs.v14i2.28

\section{Introduction}

Dentists are often the first health care professionals to diagnose a systemic disease through observation of its oral manifestations. One such disease is gastro-esophageal reflux disease (GERD), which may be evidenced by dental erosion. GERD is defined as involuntary muscle relaxing of the lower esophageal sphincter, which allows refluxed acid to move upward through the esophagus into the oral cavity. ${ }^{1}$ It is a relatively common condition worldwide, with prevalence rates in adults ranging from $21 \%$ to $56 \%$ in different countries. $^{2}$

\section{Corresponding Authors:}

Ayse Dundar

Assistant Professor, Department of Restorative

Dentistry, School of Dentistry,

University of Abant Izzet Baysal, Bolu, Turkey

Tel: + 903742705353

Fax: + 903742700066

E-mail: ayse_dent@hotmail.com

Abdulkadir Sengun

Assoc. Prof., Department of Restorative Dentistry, School of Dentistry,

University of Kirıkale, Kirıkkale, Turkey

Tel: +90 $3182244927-2243618$

Fax: +90 3182250685

E-mail: abdulkadir.sengun@kku.edu.tr
In healthy individuals, most gastric fluid is returned to the stomach by peristalsis stimulated by swallowing. The remaining fluid is cleared by secondary peristalsis stimulated by direct contact of the juice with the esophageal mucosa. ${ }^{3}$ In contrast, patients with GERD have delayed acid clearance, ${ }^{4}$ and the gastric acid and contents are involuntarily passed through the esophagus and into the oral cavity. ${ }^{5}$

The typical manifestations of GERD are heartburn, regurgitation, dysphagia, and retrosternal pain. ${ }^{6}$ Atypical manifestations, such as asthma, chronic cough, hoarseness, noncardiac chest pain, and dental erosion, are often underappreciated and poorly understood. ${ }^{7}$ Dentists are commonly the first to diagnose GERD through erosion of teeth because most people are not aware of the presence of the disease.

Dental erosion is defined as the loss of tooth substance by chemical processes (acid exposure) not involving bacteria. ${ }^{8}$ Repeated or prolonged exposure of teeth to acid leads to selective dissolution of specific components of the tooth surface, with eventual loss of tooth substance, hypersensitivity, functional impairment, and even tooth fracture. The severity of dental erosion due to GERD is related to the duration of the disease frequency of reflux, the $\mathrm{pH}$ and type of acid, and the quality and quantity of saliva.' However, if enamel demineralization is detected sufficiently early before the damage becomes irreversible, ${ }^{10,11}$ the enamel framework can be remineralized using oral regimes and preventative modifications in diet, behavior, or medication. 
This review provides an overview of some aspects of the dental approach to erosive tooth wear in GERD.

\section{Diagnostic protocol}

Early recognition of dental erosion is important to prevent serious irreversible damage to dentition. This requires awareness of the clinical appearance of erosion compared to other forms of tooth wear. An understanding of the etiologies and risk factors for erosion is also important. The aforementioned forms the basis of the diagnostic protocol and the management strategy that address the multifactorial nature of tooth wear. The primary dental care team has the expertise and the responsibility to provide this care for their patients with erosion.

To address the dental implications of GERD symptoms, the dental professional has to conduct a thorough assessment following a diagnostic protocol, which includes collecting data on the patient's medical history and dietary history, occupational / recreational history, dental history, and oral hygiene methods, in addition to an intraoral examination, head and neck examination, and salivary function examination. ${ }^{12}$

\section{Medical bistory}

The initial evaluation begins with a through medical history review, including symptoms of gastric reflux, any history of vomiting, previous investigations for gastro-intestinal complaints, and a listing of all prescription and nonprescription medications. ${ }^{13}$ Information on the use of drugs by patients is needed to help identify the etiology of any dental erosion. Some medications cause salivary hypofunction and dental erosion; drugs used to treat GERD also cause erosion. ${ }^{12}$ The acidic nature of the medication means it acts directly on the teeth. Prolonged use of 2-adrenoreceptor stimulants, such as salbutamol, salmeterol, or terbutaline, leads to decreased salivary flow, thus reducing the modifying and protective effects of saliva. ${ }^{14}$ Bronchodilatators act to relax smooth muscle and may affect the esophageal sphincter, in addition to the bronchus, and thereby potentiate the gastroesophageal reflux, which is a recognized etiological factor in tooth erosion. ${ }^{14}$ Chewable vitamin $\mathrm{C}$ tablets can cause a $\mathrm{pH}$ less than 2.0 in the oral cavity, and the $\mathrm{pH}$ of the saliva can drop while chewing vitamin $\mathrm{C}$ tablets. ${ }^{15}$
General symptoms of GERD include belching and heartburn, although some patients experience no symptoms at all. ${ }^{5}$ Dentists should evaluate patients for GERD. The evaluation should include questions about the incidence of belching, heartburn, stomachache, an acidic taste in the mouth, hoarseness, coughing, spontaneous vomiting, halitosis, choking, and excess salivation. ${ }^{16}$ If a dentist is suspicious that a patient may have gastric reflux, the dentist should refer the patient for further medical evaluation to a gastroenterologist to be evaluated for GERD.

\section{Dietary history}

It is important that the dentist obtains detailed information from patients about diet to determine the etiological factors underlying dental erosion and to implement adequate preventive measures. Erosion can be related to dietary factors. The frequency of consumption of acid drinks (soft drinks, fruit juices, sport drinks) and particular foods (citrus fruits, salad dressing) is an important factor in tooth erosion ${ }^{17}$ because they contain acids, such as citric acid, maleic acid, and phosphoric acid, ${ }^{18}$ which decrease the $\mathrm{pH}$ of the oral environment. Patients should be questioned about their consumption of acidic foods and drinks and about the frequency of their consumption.

\section{Occupational/ recreational history}

Frequent contact with acids in the workplace can increase the occurrence and/or the severity of dental erosion. Workers who had contact with acids not only showed erosion but also complained in $80 \%$ of cases about dentine hypersensitivity. ${ }^{19}$ Acid fumes at work seem to be associated with tooth surface loss, with no clinical significant difference between inorganic and organic acids. ${ }^{20}$

Wine has a low $\mathrm{pH}$ and a low content of $\mathrm{P}$ and $\mathrm{Ca}$ and therefore has erosive potential. ${ }^{21}$ Professional wine tasting is very common all over the world. In a review by Geurtsen, ${ }^{22}$ intensive swimmers showed an increased prevalence of dental erosion due to low $\mathrm{pH}$ gas-chlorinated pool water.

To determine the etiological factors of dental erosion, the dentist should obtain detailed information from the patient about his/her occupation and recreations. 
It is challenging to distinguish the influence of abrasion or erosion and to judge the activity and progression of dental erosion. Color changes and sensitivity may provide some information about lesions. ${ }^{23}$ Typical signs of enamel erosion on buccal and lingual sites are the appearance of a smooth, silky-glazed, sometimes dull, enamel surface with the absence of perikymata, together with intact enamel along the gingival margin. ${ }^{23}$ Eroded teeth have the appearance of having been lightly prepared for fullcoverage restorations with a chamfer margin and are more prone to wear. ${ }^{4}$ It has been hypothesized that a preserved enamel band along oral and facial gingival margins could be due to plaque remnants, which could act as a diffusion barrier to acids. ${ }^{23}$ This phenomenon could also be due to an acid-neutralizing effect of the sulcular fluid, which has a $\mathrm{pH}$ between 7.5 and 8.0. ${ }^{23}$ In more advanced stages of dental erosion, changes in the morphology result in a concavity in the enamel, the width of which clearly exceeds its depth. ${ }^{23}$ Further progression of occlusal erosion leads to a rounding of the cusps and restorations rising above the level of the adjacent tooth surfaces. ${ }^{23}$ In severe cases, the whole occlusal morphology disappears, ${ }^{23}$ and pulp exposure may occur.

Erosive tooth wear can involve any surface of the teeth, but it is most commonly seen on the facial, occlusal, and lingual surfaces. ${ }^{24}$ Thinning of the enamel imparts an unaesthetic yellowish hue to the teeth. ${ }^{4}$ Once dentin is exposed, the loss of dentin progresses faster than the loss of enamel, such that "cupping" of lesions on the occlusal surfaces occurs. ${ }^{25}$ Amalgam restorations in eroded teeth appear highly polished and seem to "stand above" the tooth surface. ${ }^{4}$

Extrinsic erosion from the ingestion of acidic foods and beverages occurs primarily on the labial surfaces of anterior teeth, the buccal surfaces of posterior teeth, and the occlusal surfaces of molar teeth. ${ }^{16}$ Intrinsic erosion (bulimia and GERD) primarily occurs on the palatal surfaces of maxillary teeth and the occlusal surfaces of the mandibular molars. ${ }^{16}$ Dental erosions on the lingual surfaces of anterior maxillary teeth have been noted in bulimic patients. ${ }^{26}$ Those of the posterior teeth involving palatinal/lingual and occlusal surface may suggest GERD. ${ }^{27}$

It is recognized that refluxed acid first attacks the palatal surfaces of the upper incisor teeth. ${ }^{28,29}$ Later, if the condition continues, erosion of the occlusal surfaces of the posterior teeth in both arches and the labial or buccal surfaces results from the extended period of acid reflux..$^{28,29}$

\section{Dental history}

During intraoral examinations of patients, restorations are a clue for dentists. The tooth structure surrounding the restorations dissolves much more rapidly than the restorative material. ${ }^{16}$

\section{Salivary function}

The importance of saliva in clearing and neutralizing acids from the dental surface has been confirmed. ${ }^{30}$ Saliva contains buffers to resist changes in $\mathrm{pH}$ and provides a constant supply of ions to the tooth surface. ${ }^{31}$ Saliva, with its buffering capacity and its ability to form a protective enamel pellicle, can control dental decalcification. ${ }^{32}$ Once the erosive agent is neutralized and/or cleared from the tooth surface, the deposition of salivary calcium and phosphate can lead to remineralization of some of the softened tissue. ${ }^{30}$ Any reduction in the loss of the salivary buffering capacity contributes to the process of enamel erosion. ${ }^{33}$

Assessing salivary parameters, such as $\mathrm{pH}$ and buffering capacity, in the dental office is possible using commercially available diagnostic kits. The buffering capacity and, if desired, the $\mathrm{pH}$ of the saliva can be measured in freshly collected saliva using an indicator system. ${ }^{34}$ The flow rate and the buffering capacity of saliva are increased by chewing. ${ }^{35}$ Saliva buffers the acid within the distal esophagus, and swallowing increases the rate of peristalis; both are recognized as major factors in esophageal acid clearance. ${ }^{13}$

\section{Management of erosive tooth wear in GERD}

\section{Identiffing the source of the erosive tooth wear}

The pattern and the location of erosive tooth wear often provide major clues as to the cause. Extrinsic erosion from the ingestion of acidic foods and beverages occurs primarily on the labial surfaces of anterior teeth, the buccal surfaces of posterior teeth, and the occlusal surfaces of molar teeth. ${ }^{13}$ Intrinsic erosion (bulimia and GERD) primarily occurs on the palatal surfaces of maxillary teeth and the occlusal surfaces of the mandibular molars. ${ }^{13}$ Suspected GERD patients should be referred to a gastroenterologist for definitive diagnosis and treatment. 
Dentists and patients should be aware of acidic attack that may cause irreversible damage to dental hard tissue. First, reducing the level of oral acidity will prevent erosive attacks. For those at high risk of dental erosion, it is advisable to consume in moderation by decreasing the frequency of ingestion of potentially harmful drinks and food, restricting them to main meals, and minimizing their contact time with teeth. ${ }^{18,30}$, ${ }^{31}$ Frequent chewing of sugar-free antacid medications will help to reduce the acidity and to improve salivary flow rates. ${ }^{16}$ Patients can take antacids immediately after heartburn or a sensation of acid reflux into the oral cavity. ${ }^{36}$ Patients with erosion should be educated to rinse the mouth with water, sodium bicarbonate, and a fluoride mouthwash immediately after a significant acid challenge. ${ }^{16}$ The water will eliminate most of the acid, the sodium bicarbonate will neutralize the remaining acid, and the fluoride mouthwash will help remineralize the etched tooth structure. ${ }^{16}$ They must be informed not to brush their teeth immediately after an acid challenge and not to use strongly abrasive toothpastes. ${ }^{34}$ To prevent the acidity on the surface of the teeth from falling below $\mathrm{pH} 5.5$, at which point demineralization occurs, the consumption of carbonated and acidic beverages must be curtailed. ${ }^{4}$

The action of fluoride is mainly attributed to a precipitation of CaF-like material on eroded dental surfaces. ${ }^{37}$ High-concentrated fluoride agents, such as oral rinses, gels, or varnishes, have been demonstrated to increase abrasion resistance and decrease the development of enamel and dentin erosion in vitro and in situ. ${ }^{37,38}$ Fluoride varnish should be applied every three months. Prescription fluoride toothpaste should be recommended for daily use.

The protective effects of laser application on enamel and dentin demineralization have gained increasing attention in recent years. Laser application melts and solidifies the dental surface, creating a new, smoother surface. ${ }^{39}$ In recent years, casein phosphopeptide/amorphous calcium phosphate (CPP-ACP) has been used to reduce dental erosion. Amorphous calcium phosphate should be applied nightly by rubbing it on the teeth with a finger or using a bleaching tray. ${ }^{16}$

The protection of dentin from erosion and abrasion with resin-based dentine bonding agents has also been reported. ${ }^{40}$ The application of dentine bonding agents was found to be an effective

preservative treatment option in the management of dentin hypersensitivity. ${ }^{41}$ It might be reasonable to use a close fitting occlusal guard during times of high risk of erosive challenge, such as during sleep (for patients with reflux). ${ }^{42}$

Patients with reduced salivary flow rates are especially vulnerable to dental erosion. Patients with GERD are also frequently dehydrated and have reduced salivary flow rates. ${ }^{16}$ To improve salivary flow rates, patients suffering from erosion should be advised to use sugar-free antacids and xylitol chewing gum. ${ }^{36}$ Xylitol promotes mineralization by increasing the flow of saliva and by inhibiting the growth, metabolism, and polysaccharide production of Streptococci mutans. ${ }^{43}$ Patients with dental erosion should be instructed to chew a xylitol-containing chewing gum immediately after every meal for 5 minutes and after major acid challenges. Prescribing pilocarpine in consultation with a physician may be considered in xerostomic patients to improve salivary flow rates. ${ }^{16}$ If improving salivary flow rates is an important strategy for a patient, it is very useful to obtain baseline data on flow rates before initiating therapy. A kit is available to determine stimulated flow rates, salivary $\mathrm{pH}$, and buffering capacity.

\section{Restorative treatment}

Patients suffering from chronic regurgitation and heartburn symptoms for two or more days a week should be referred to a physician for further investigation, as the untreated condition can lead to complications, including erosive esophagitis, stricture formation, and Barrett's esophagus, which increase the risk of esophageal adenocarcinoma. ${ }^{44}$

Some patients may not be aware of their condition as a disease or disorder until it starts affecting their teeth. These patients should be referred to an appropriate specialist ${ }^{42}$ or an interdisciplinary team.

The dentist should consider restorative treatment when: (1) the structural integrity of the affected teeth is threatened; (2) the teeth are hypersensitive; (3) there is significant loss of tooth structure, vertical dimension, and/or function; (4) the defect is esthetically unacceptable to the patient; and (5) pulpal exposure is likely to occur. ${ }^{45}$

Initially, erosive tooth wear is limited to the enamel. At this stage of the erosive process, the teeth are not hypersensitive. Restorations may be inserted because of esthetic needs and/or to prevent further progression. Direct composite coatings should be 
considered as the treatment of choice, or porcelain veneers in more advanced cases. This seals the enamel and reestablishes the tooth contour and decreases further enamel loss by acid exposure.

In patients with severe tooth surface loss on more than two surfaces per tooth and extended loss of vertical dimension, complex reconstruction with indirect restorations (ceramic crowns, bridges) is often inevitable. ${ }^{46}$

\section{Recommendations for patients with GERD}

- Be examined by a gastroenterologist for a definitive diagnosis and treatment.

- Take antacids immediately after heartburn or sensation of acid reflux into the oral cavity

- Rinse the mouth with water, sodium bicarbonate, and a fluoride mouthwash immediately after a significant acid challenge

- Avoid brushing teeth immediately after an acid challenge and using strongly abrasive toothpastes

- Decrease the consumption of carbonated and acidic beverages

- Use xylitol chewing gum to stimulate salivary flow

- Use a close fitting occlusal guard during times of high risk of erosive challenge, such as during sleep

- Use desensitizing and fluoride toothpaste

\section{References}

1. Bartlett DW, Evans DF, Smith BG. Oral regurgitation after reflux provoking meals: a possible cause of dental erosion? Journal of Oral Rehabilitation 1997; 24: 102-108.

2. Broliato GA, Volcato DB, Reston EG, Kramer PF, Marquezan M, Ruzzarin F. Esthetic and functional dental rehabilitation in a paient with gastroesophageal reflux. Quintessence International 2008; 39: 131-137.

3. Kruse-Anderson S, Wallin L, Madsen T. Acid gastro-oesophageal reflux and oesophageal pressure activity during postprandial and nocturnal periods. A study in subjects with and without pathologic acid gastrooesophageal reflux. Scandinavian Journal of Gastroenterology 1987; 22: 926-930.
4. Barron RP, Carmichael RP, Marcon MA, Sandor GK. Dental erosion in gastroesophageal reflux disease. Journal of the Canadian Dental Association 2003; 69: 8489.

5. Liberali S. Oral impact of gastro-oesophageal reflux disease: a case report. Australian Dental Journal 2008; 53: 176-179.

6. Anggiansah A, Owen WA, Owen WJ. The investigation and management of gastro-oesophageal reflux disease. The British Journal of Clinical Practice 1993; 47: 256-261.

7. Chandra A, Moazzez R, Bartlett D, Anggiansah A, Owen WJ. A review of the atypical manifestations of gastroesophageal reflux disease. International Journal of Clinical Practice 2004; 58: 41-48.

8. Lussi A. Erosive tooth wear - a multifactorial condition of growing concern and increasing knowledge. Monographs in Oral Scieence 2006; 20: 1-8.

9. Su JM, Tsamtsouris A, Laskou M. Gastroesophageal reflux in children with cerebral palsy and its relationship to erosion of primary and permanent teeth. Journal of the Massachusetts Dental Society 2003; 52: 20-24.

10. Mandel ID. Caries prevention: current strategies, new directions. Journal of American Dental Association 1996; 127: 1477-1488.

11. Geiger S, Matalon S, Blasbalg J, Tung M, Eichmiller FC. The clinical effect of amorphous calcium phosphate (ACP) on root surface hypersensitivity. Operative Dentistry 2003; 28: 496-500.

12. Gandara BK, Truelove EL. Diagnosis and management of dental erosion. Journal of Contemporary Dental Practice 1999; 1: 16-23.

13. Barrow SY. Is your knowledge up-to-date? Int J Dent Hyg. 2010; 8: 319-323.

14. Hellwig E, Lussi A. Oral hygiene products and acidic medicines. Monographs in Oral Science 2006; 20: 112-118.

15. Giunta JL. Dental erosion resulting from chewable vitamin C tablets. Journal of American Dental Association 1983; 107: 253-256.

16. Donovan T. Dental erosion. Journal of Esthetic and Restorative Dentistry 2009; 21: 359-364.

17. Magalhaes AC, Wiegand A, Rios D, Honorio HM, Buzalaf MA. Insights into preventive measures for dental erosion. Journal of Applied Oral Science 2009; 17 : 75-86.

18. Lussi A, Jaeggi T, Zero D. The role of diet in the aetiology of dental erosion. Caries Research 2004; 38 Suppl 1: 34-44.

19. Amin WM, Al-Omoush SA, Hattab FN. Oral health 
status of workers exposed to acid fumes in phosphate and battery industries in Jordan. International Dental Journal 2001; 51: 169-174.

20. Lussi A, Jaeggi T. Occupation and sports. Monographs in Oral Science 2006; 20: 106-111.

21. Lussi A, Jaeggi T. Chemical factors. Monographs in Oral Science 2006; 20: 77-87.

22. Geurtsen W. Rapid general dental erosion by gaschlorinated swimming pool water. Review of the literature and case report. American Journal of Dentistry 2000; 13: 291-293.

23. Lussi A, Jaeggi T. Erosion--diagnosis and risk factors. Clinical Oral Investigation 2008; 12 (Suppl): 5-13.

24. Lazarchik DA, Filler SJ. Effects of gastroesophageal reflux on the oral cavity. American Journal of Medicine 1997; 103: 107-113.

25. Lazarchik DA, Filler SJ. Dental erosion: predominant oral lesion in gastroesophageal reflux disease. American Journal of Gastroenteroloy 2000; 95 (8 Suppl): 33-38.

26. Jones RR, Cleaton-Jones P. Depth and area of dental erosions, and dental caries, in bulimic women. Journal of Dental Research 1989; 68: 1275-1278.

27. Schroeder PL, Filler SJ, Ramirez B, Lazarchik DA, Vaezi MF, Richter JE. Dental erosion and acid reflux disease. Annals of Internal Medicine 1995; 122: 809815.

28. Bartlett DW, Evans DF, Smith BG. The relationship between gastro-oesophageal reflux disease and dental erosion. Journal of Oral Rehabilitation 1996; 23: 289297.

29. Spigset O. Oral symptoms in bulimia nervosa. A survey of 34 cases. Acta Odontoligica Scandinavica 1991; 49: 335-339.

30. Lussi A, Hellwig E. Risk assessment and preventive measures. Monographs in Oral Science 2006; 20: 190199.

31. Imfeld T. Dental erosion. Definition, classification and links. Europen Journal of Oral Science 1996; 104: 151-155.

32. Meurman JH, Vesterinen M. Wine, alcohol, and oral health, with special emphasis on dental erosion. Quintessence International 2000; 31: 729-733.

33. Guldag MU, Buyukkaplan US, Ay ZY, Katirci G. A multidisciplinary approach to dental erosion: a case report. Europen Journal of Dentistry 2008; 2: 110-114.
34. Lussi A. Dental erosion clinical diagnosis and case history taking. Europen Journal of Oral Science 1996; 104: 191-198.

35. Edgar WM. Saliva and dental health. Clinical implications of saliva: report of a consensus meeting. Brazilian Dental Journal 1990; 169: 96-98.

36. Farrokhi F, Vaezi MF. Extra-esophageal manifestations of gastroesophageal reflux. Oral Disease 2007; 13: 349-359.

37. Ganss C, Klimek J, Brune V, Schurmann A. Effects of two fluoridation measures on erosion progression in human enamel and dentine in situ. Caries Research 2004; 38: 561-566.

38. Lagerweij MD, Buchalla W, Kohnke S, Becker K, Lennon AM, Attin T. Prevention of erosion and abrasion by a high fluoride concentration gel applied at high frequencies. Caries Research 2006; 40: 148-153.

39. Nelson DG, Wefel JS, Jongebloed WL, Featherstone JD. Morphology, histology and crystallography of human dental enamel treated with pulsed low-energy infrared laser radiation. Caries Research 1987; 21: 411-426. 40. Azzopardi A, Bartlett DW, Watson TF, Sherriff M. The surface effects of erosion and abrasion on dentine with and without a protective layer. Brazilian Dental Journal 2004; 196: 351-354.

41. Brunton PA, Kalsi KS, Watts DC, Wilson NH. Resistance of two dentin-bonding agents and a dentin densensitizer to acid erosion in vitro. Dental Materials 2000; 16: 351-355.

42. Amaechi BT, Higham SM. Dental erosion: possible approaches to prevention and control. Journal of Dentistry 2005; 33: 243-252.

43. Soderling EM. Xylitol, mutans streptococci, and dental plaque. Advances in Dental Research 2009; 21: 74-78.

44. Howden CW, Chey WD. Gastroesophageal reflux disease. The Journal of Family Practice 2003; 52: 240247.

45. Lambrechts P, Van Meerbeek B, Perdigao J, Gladys S, Braem M, Vanherle G. Restorative therapy for erosive lesions. Europen Journal of Oral Science 1996; 104: 229-240.

46. Jaeggi T, Gruninger A, Lussi A. Restorative therapy of erosion. Monographs in Oral Science 2006; 20: 200214. 